\title{
A NOTE ON SIMULTANEOUS BLOCK DIAGONALLY STABLE MATRICES*
}

\author{
I. BRÁS ${ }^{\dagger}$, A.C. CARAPITO ${ }^{\ddagger}$, AND P. ROCHA ${ }^{\S}$
}

\begin{abstract}
Consider a set of square real matrices of the same size $\mathcal{A}=\left\{A_{1}, A_{2}, \ldots, A_{N}\right\}$, where each matrix is partitioned, in the same way, into blocks such that the diagonal ones are square matrices. Under the assumption that the diagonal blocks in the same position have a common Lyapunov solution, sufficient conditions for the existence of a common Lyapunov solution with block diagonal structure for $\mathcal{A}$ are presented. Furthermore, as a by-product, an algorithm for the construction of such a common Lyapunov solution is proposed.
\end{abstract}

Key words. Diagonal stability, Lyapunov matrix inequalities, Block matrices.

AMS subject classifications. 15A24, 15A42, 93D05.

1. Introduction. Consider a finite set $\mathcal{A}=\left\{A_{1}, \ldots, A_{N}\right\}$ of matrices in $\mathbb{R}^{n \times n}$, $\mathcal{A}$ is said to be simultaneously stable if there exists a positive definite and symmetric matrix $P$ which is a Lyapunov solution for every $A_{p} \in \mathcal{A}$, i.e., if there exists $P$ such that $\mathcal{L}_{A_{p}}(P):=-\left(A_{p}^{T} P+P A_{p}\right)$ is positive definite, for every $A_{p} \in \mathcal{A}$. The matrix $P$ is called a common Lyapunov solution (CLS) for $\mathcal{A}$, [1]. In practice, simultaneously stable sets of matrices play an important role, for instance, in the study of the stability of a linear switched system. Switched systems are a class of hybrid dynamical systems consisting of a family of linear time invariant systems $\Sigma_{p}: \dot{x}(t)=A_{p} x(t)$ and a rule $\sigma$ (switching signal) that supervises the switching among them. More precisely, associated with $\mathcal{A}$ the following switched linear system may be defined

$$
\Sigma: \dot{x}(t)=A_{\sigma(t)} x(t),
$$

where $x(t) \in \mathbb{R}^{n}$ is the state and $\sigma:[0,+\infty[\rightarrow \mathcal{P}$, with $\mathcal{P}=\{1, \ldots, N\}$, is a piecewise constant function. For more details see for instance [11, 13, 17]. If $P$ is a common Lyapunov solution for $\mathcal{A}$, then the systems $\Sigma_{p}$ share a common quadratic Lyapunov function (CQLF) $V(x)=x^{T} P x$, and, therefore, the switched system $\Sigma$ is uniformly

${ }^{*}$ Received by the editors on August 1, 2012. Accepted for publication on January 21, 2013. Handling Editor: Bryan L. Shader.

${ }^{\dagger}$ CIDMA - Center for Research and Development in Mathematics and Applications, Department of Mathematics, University of Aveiro, Portugal (ibras@ua.pt).

$\ddagger$ CIDMA - Center for Research and Development in Mathematics and Applications, Department of Mathematics, University of Beira Interior, Portugal (carapito@ubi.pt).

${ }^{\S}$ CIDMA - Center for Research and Development in Mathematics and Applications, Faculty of Engineering, University of Porto, Portugal (mprocha@fe.up.pt). 
exponentially stable, $[11,13,17]$. Systems that admit this type of Lyapunov functions are commonly called quadratic Lyapunov stable. Other examples of applications may be found in $[9]$.

The common Lyapunov solution existence problem (also known as the CQLF existence problem) has been widely studied and it is known to be very hard to tackle in general in a simple algebraic manner. However, in some cases conditions are known that guarantee the existence of a CLS; a very good survey about this may be found in [17]. Despite the large amount of work that has been done on this subject, the CLS existence problem is still challenging and and keeps attracting the attention of several researchers, see for instance $[4,7,10]$.

If there exists a diagonal matrix $D$ such that $A^{T} D+D A<0$, the matrix $A$ is said to be diagonally stable, see for instance [9]. A set of matrices is said to be simultaneous diagonally stable if it admits a diagonal CLS. Diagonal stability has been used in several applications, see [9], for a good survey, and, for more recent research on this notion; see for instance $[2,4,15]$.

In this paper, we address the problem of the existence of a common Lyapunov solution using an analysis in terms of block matrices. More precisely, we investigate the existence of a CLS with pre-specified block diagonal structure for an arbitrary set of stable matrices. Notice that some efforts have already made for the particular case where the matrices are block triangular, see for instance [5]. This kind of block analysis arises quite naturally when analyzing the stability of the some types of switched systems interconnections, [3].

The paper is organized as follows. In Section 2, notations, basic definitions and preliminary results are presented. In Section 3, the property of block simultaneous diagonal stability for a collection of matrices partitioned into $2 \times 2$ blocks is analysed. Section 4 extends the study done in Section 3 for matrices partitioned into $r \times r$ blocks, $r \geq 2$. Also, in Section 4 , an algorithm and numerical examples are presented to illustrate the obtained results. Finally, last section is devoted to the conclusions.

2. Preliminaries. Let $\mathcal{A}=\left\{A_{1}, \ldots, A_{N}\right\}$ be a set of matrices in $\mathbb{R}^{n \times n}$. Consider that each matrix $A_{p} \in \mathcal{A}$ is similarly partitioned into $r \times r$ blocks as follows:

$$
A_{p}=\left[\begin{array}{cccc}
A_{11}^{p} & A_{12}^{p} & \cdots & A_{1 r}^{p} \\
A_{21}^{p} & A_{22}^{p} & \cdots & A_{2 r}^{p} \\
\vdots & \vdots & \ddots & \vdots \\
A_{r 1}^{p} & A_{r 2}^{p} & \cdots & A_{r r}^{p}
\end{array}\right]
$$

where $A_{i i}^{p} \in \mathbb{R}^{n_{i} \times n_{i}}$, for each $p=1,2, \ldots, N$ and $n_{1}+\cdots+n_{r}=n$. Here and throughout the text the upper index $p$ should not be mistaken for an exponent. 
For short we say that $A_{p}$ is an $r$-block matrix. The set $\mathcal{A}$ is said to be $r$-block simultaneous diagonally stable, if there exists a CLS for $\mathcal{A}$ with a compatible block diagonal structure. That is, if there exists $P=\operatorname{diag}\left(P_{1}, \ldots, P_{r}\right)$, with $P_{i} \in \mathbb{R}^{n_{i} \times n_{i}}$ positive definite matrices, such that $\mathcal{L}_{A_{p}}(P):=-\left(A_{p}^{T} P+P A_{p}\right)$ is positive definite, for all $p=1,2, \ldots, N$.

We begin by establishing a necessary condition for a set $\mathcal{A}$ of $r$-block matrices to be $r$-block simultaneous diagonally stable. For each $i$, we denote by $\mathcal{A}_{i}$ the set of blocks $(i, i)$ of all matrices of $\mathcal{A}$, that is, $\mathcal{A}_{i}=\left\{A_{i i}^{1}, \ldots, A_{i i}^{N}\right\}$, for $i \in\{1, \ldots, r\}$. It is not difficult to show that:

LEMMA 2.1. If $\mathcal{A}$ is a r-block simultaneous diagonally stable set then the sets $\mathcal{A}_{1}, \ldots, \mathcal{A}_{r}$ are simultaneously stable.

The previous lemma becomes a necessary and sufficient condition in the block triangular case (lower or upper), see [5].

TheOREm 2.2. A set $\mathcal{A}$ of $r$-block upper triangular matrices is $r$-block simultaneous diagonally stable if and only if the sets $\mathcal{A}_{1}, \ldots, \mathcal{A}_{r}$ of diagonal blocks are simultaneously stable.

The proof of Theorem 2.2 presented in [5] consists of showing that, in the block triangular case, it is always possible to construct a $r$-block diagonal CLS for $\mathcal{A}$ from a set $P_{1}, P_{2}, \ldots, P_{r}$ of CLSs for $\mathcal{A}_{1}, \mathcal{A}_{2}, \ldots, \mathcal{A}_{r}$, respectively. Following this approach, our aim is to study the existence and construction of a CLS with block diagonal structure for a collection $\mathcal{A}$ of matrices with a pre-specified $r$-block structure, as defined in (2.1), starting from such a set of CLSs, without assuming the block triangularity.

Throughout the text, $\mathcal{P}=\{1, \ldots, N\}$ denotes a finite index set, $\lambda(\cdot), \lambda_{\max }(\cdot)$ and $\lambda_{\min }(\cdot)$ are used to denote an eigenvalue, the largest eigenvalue and the smallest eigenvalue of a symmetric matrix and for a real symmetric matrix, $S>0$ denotes that $S$ is a positive definite matrix and $S^{T}$ denotes the transpose of $S$.

3. The 2-block case. For sake of simplicity, in this section we consider a set $\mathcal{A}=\left\{A_{p}: p \in \mathcal{P}\right\}$ of matrices partitioned into $2 \times 2$ blocks of the form

$$
A_{p}=\left[\begin{array}{ll}
A_{11}^{p} & A_{12}^{p} \\
A_{21}^{p} & A_{22}^{p}
\end{array}\right], p \in \mathcal{P}
$$

and assume that there exists a CLS $P_{i}$ for $\mathcal{A}_{i}=\left\{A_{i i}^{p}: p \in \mathcal{P}\right\}, i=1,2$. Following the approach used in [12] for the construction of a diagonal CLS for a set of stable triangular (non-block) matrices, we start by considering two basic problems.

Problem 3.1. Establish conditions on the matrices $A_{p}, P_{1}$ and $P_{2}$, that guarantee the existence of an $\epsilon>0$ such that $P=\operatorname{diag}\left(P_{1}, \epsilon P_{2}\right)$ is a CLS for $\mathcal{A}$. 
Note that the existence of such an $\epsilon$ is equivalent to the positive definiteness of the following matrices:

$$
M^{p}(\epsilon):=\left[\begin{array}{cc}
Q_{1}^{p} & \epsilon\left(A_{21}^{p}\right)^{T} P_{2}+P_{1} A_{12}^{p} \\
\epsilon P_{2} A_{21}^{p}+\left(A_{12}^{p}\right)^{T} P_{1} & \epsilon Q_{2}^{p}
\end{array}\right], p \in \mathcal{P},
$$

where $Q_{1}^{p}:=\mathcal{L}_{A_{11}^{p}}\left(P_{1}\right)>0$ and $Q_{2}^{p}:=\mathcal{L}_{A_{22}^{p}}\left(P_{2}\right)>0$.

To deal with Problem 1, we begin by analyzing the existence of a Lyapunov solution $P=\operatorname{diag}\left(P_{1}, \epsilon P_{2}\right)$ for a single matrix in $\mathcal{A}$, where $P_{1}$ and $P_{2}$ are known to be Lyapunov solutions for the blocks in its diagonal, respectively, and $\epsilon>0$ is to be determined. From this, we formulate the following problem.

Problem 3.2. Given a matrix $A=\left[\begin{array}{ll}A_{11} & A_{12} \\ A_{21} & A_{22}\end{array}\right]$, for which $P_{1}$ and $P_{2}$ are Lyapunov solutions of $A_{11}$ and $A_{22}$, respectively, find $\varepsilon>0$ such that $P=\operatorname{diag}\left(P_{1}, \epsilon P_{2}\right)$ is a Lyapunov solution for $A$.

The following lemma gives two conditions (a necessary condition and a sufficient one) for the existence of such an $\varepsilon$. The proof of the sufficient condition yields an interval of variation for $\varepsilon$.

TheOREM 3.3. Let $A=\left[A_{i j}\right]$ be a 2-block matrix such that $P_{1}$ and $P_{2}$ are Lyapunov solutions for $A_{11}$ and $A_{22}$. Consider $U:=A_{12}^{T} P_{1}, V:=P_{2} A_{21}, S:=$ $V Q_{1}^{-1} V^{T}, R:=U Q_{1}^{-1} U^{T}$ and $L:=Q_{2}-U Q_{1}^{-1} V^{T}-V Q_{1}^{-1} U^{T}$, where $Q_{1}=\mathcal{L}_{A_{11}}\left(P_{1}\right)$ and $Q_{2}=\mathcal{L}_{A_{22}}\left(P_{2}\right)$. The following statements hold:

(1) If there exists $\epsilon>0$ such that $P=\operatorname{diag}\left(P_{1}, \epsilon P_{2}\right)$ is a Lyapunov solution for $A$, then $\lambda_{\min }(L)>2\left(\lambda_{\min }(R) \lambda_{\min }(S)\right)^{\frac{1}{2}}$

(2) If $\lambda_{\min }(L)>2\left(\lambda_{\max }(R) \lambda_{\max }(S)\right)^{\frac{1}{2}}$, then there exists $\epsilon>0$ such that $P=$ $\operatorname{diag}\left(P_{1}, \epsilon P_{2}\right)$ is a Lyapunov solution for $A$.

Proof. The matrix $P=\operatorname{diag}\left(P_{1}, \epsilon P_{2}\right)$ is a Lyapunov solution for $A$ if and only if

$$
M(\epsilon):=\left[\begin{array}{cc}
Q_{1} & \epsilon A_{21}^{T} P_{2}+P_{1} A_{12} \\
\epsilon P_{2} A_{21}+A_{12}^{T} P_{1} & \epsilon Q_{2}
\end{array}\right]>0 .
$$

Using the Schur Complement of the block $Q_{1}$ on $M(\epsilon)$, we conclude that $M(\epsilon)>0$ if and only if

$$
\epsilon Q_{2}-(\epsilon V+U) Q_{1}^{-1}\left(\epsilon V^{T}+U^{T}\right)>0
$$

After an easy but cumbersome computation we conclude that, $P$ is Lyapunov solution for $A$ if and only if

$$
-\epsilon^{2} S+\epsilon L-R>0 .
$$


Proof of (1). If there exists $\epsilon>0$ such that $P=\operatorname{diag}\left(P_{1}, \epsilon P_{2}\right)$ is a Lyapunov solution for $A$ then, it follows from (3.1) that $\epsilon L>\epsilon^{2} S+R$ and, because $R \geq 0$ and $S \geq 0$, then $L>0$. Therefore, taking into account the positive definite ordering [8, p. 471], it follows that

$$
\epsilon \lambda_{\min }(L)>\lambda_{\min }\left(\epsilon^{2} S+R\right) .
$$

Since, by Weyl's theorem [8, p. 181], $\lambda_{\min }\left(\epsilon^{2} S+R\right)>\epsilon^{2} \lambda_{\min }(S)+\lambda_{\min }(R)$, we obtain from $(3.2)$

$$
\epsilon \lambda_{\min }(L)>\epsilon^{2} \lambda_{\min }(S)+\lambda_{\min }(R)
$$

meaning that the quadratic inequality $\lambda_{\min }(S) x^{2}-\lambda_{\min }(L) x+\lambda_{\min }(R)<0$ has a real solution $(\epsilon)$. Consequently, $\lambda_{\min }^{2}(L)-4 \lambda_{\min }(R) \lambda_{\min }(S)>0$, i.e., $\lambda_{\min }(L)>$ $2\left(\lambda_{\min }(R) \lambda_{\min }(S)\right)^{\frac{1}{2}}$.

Proof of (2). The condition (3.1) is satisfied if and only if $\lambda_{\min }\left(-\epsilon^{2} S+\epsilon L-R\right)>$ 0 . By Weyl's theorem, this holds when $\epsilon \lambda_{\min }(L)+\lambda_{\min }\left(-\epsilon^{2} S-R\right)>0$. Again by Weyl's theorem, this holds when $\epsilon \lambda_{\min }(L)+\epsilon^{2} \lambda_{\min }(-S)+\lambda_{\min }(-R)>0$, or, equivalently, if

$$
-\epsilon^{2} \lambda_{\max }(S)+\epsilon \lambda_{\min }(L)-\lambda_{\max }(R)>0 .
$$

Notice that, the hypothesis of condition (2) implies that $\lambda_{\min }(L)>0$. The choice of the scalar $\epsilon$ is made considering the following different cases. If $A$ is block upper triangular matrix $(S=0)$ then, $(3.3)$ is satisfied if and only if $\epsilon \in J:=] \frac{\lambda_{\max }(R)}{\lambda_{\min }(L)},+\infty[$. If $A$ is not a block upper triangular matrix, then $S \neq 0$. Since by hypothesis

$$
\Delta:=\lambda_{\min }(L)^{2}-4 \lambda_{\max }(R) \lambda_{\max }(S)>0,
$$

clearly (3.3) is satisfied if and only if $\epsilon \in J=] \frac{\lambda_{\min }(L)-\sqrt{\Delta}}{2 \lambda_{\max }(S)}, \frac{\lambda_{\min }(L)+\sqrt{\Delta}}{2 \lambda_{\max }(S)}[$.

REMARK 3.4. Let us consider $A_{i j}, i, j=1,2$, to be stable blocks of order 1 , i.e, $A=\left[\begin{array}{ll}a_{11} & a_{12} \\ a_{21} & a_{22}\end{array}\right]$ with $a_{11}<0$ and $a_{22}<0$. In this case, the conditions in (1) and (2) of Theorem 3.3 coincide. Moreover, taking $P_{1}=P_{2}=1$ we obtain $Q_{1}=-2 a_{11}$ and $Q_{2}=-2 a_{22}$. Hence, $U=a_{12}, V=a_{21}$ and $S=-\frac{a_{21}^{2}}{2 a_{11}}, R=-\frac{a_{12}^{2}}{2 a_{11}}$ and $L=-2 a_{22}+\frac{a_{21} a_{21}}{a_{11}}$. Then, the condition in (2) becomes $-2 a_{22}+\frac{a_{21} a_{21}}{a_{11}}>\left|\frac{a_{21} a_{12}}{a_{11}}\right|$. This is equivalent to $\operatorname{det}(A)>0$, under the condition $a_{11}<0 \wedge a_{22}<0$. Note that the positiveness of the matrix determinant together with the negativity of the diagonal elements is exactly the classical necessary and sufficient conditions for the diagonal stability of a $2 \times 2$ matrix [6]. 
A solution of Problem 2 may be obtained from Theorem 3.3. Suppose that, for each matrix $A_{p}=\left[\begin{array}{cc}A_{11}^{p} & A_{12}^{p} \\ A_{21}^{p} & A_{22}^{p}\end{array}\right]$ in $\mathcal{A}$, there exist Lyapunov solutions $P_{1}$ and $P_{2}$ of $\mathcal{A}_{1}=\left\{A_{11}^{p}: p \in \mathcal{P}\right\}$ and $\mathcal{A}_{2}=\left\{A_{22}^{p}: p \in \mathcal{P}\right\}$, respectively, such that condition (3.4) is satisfied. Therefore, for each $p \in \mathcal{P}$, an interval $J_{p}$ may be identified, as shown in the proof (of (2)) of the mentioned theorem. If $\bigcap_{p \in \mathcal{P}} J_{p} \neq \emptyset$, there exists $\epsilon \in \bigcap_{p \in \mathcal{P}} J_{p}$ such that $P=\operatorname{diag}\left(P_{1}, \epsilon P_{2}\right)$ is a CLS for $\mathcal{A}$.

More precisely, let us consider the following notation:

$$
\begin{aligned}
\sigma_{p} & :=\lambda_{\max }\left(V^{p}\left(Q_{1}^{p}\right)^{-1}\left(V^{p}\right)^{T}\right) \\
\rho_{p} & :=\lambda_{\max }\left(U^{p}\left(Q_{1}^{p}\right)^{-1}\left(U^{p}\right)^{T}\right) \\
\lambda_{p} & :=\lambda_{\min }\left(Q_{2}^{p}-U^{p}\left(Q_{1}^{p}\right)^{-1}\left(V^{p}\right)^{T}-V^{p}\left(Q_{1}^{p}\right)^{-1}\left(U^{p}\right)^{T}\right)
\end{aligned}
$$

and

$$
\Delta_{p}:=\lambda_{p}^{2}-4 \rho_{p} \sigma_{p}
$$

where

$$
U^{p}:=\left(A_{12}^{p}\right)^{T} P_{1}, V^{p}:=P_{2} A_{21}^{p}, Q_{1}^{p}=\mathcal{L}_{A_{11}^{p}}\left(P_{1}\right) \text { and } Q_{2}^{p}=\mathcal{L}_{A_{22}^{p}}\left(P_{2}\right) .
$$

With this notation, considering the previous arguments, we state the following sufficient condition for the existence of a solution of Problem 1.

Corollary 3.5. Let $\mathcal{A}=\left\{A_{p}=\left[A_{i j}^{p}\right]: i, j=1,2\right.$ and $\left.p \in \mathcal{P}\right\}$ be a set of 2-block matrices such that $P_{1}$ and $P_{2}$ are CLSs for the sets of diagonal blocks $\mathcal{A}_{1}$ and $\mathcal{A}_{2}$, respectively. Then, $\mathcal{A}$ is 2-block simultaneous diagonally stable if both of the following conditions hold.

(1) $\Delta_{p}>0$, for all $p \in \mathcal{P}$.

(2) $\bigcap_{p \in \mathcal{P}} J_{p} \neq \emptyset$ where, for each $p$,

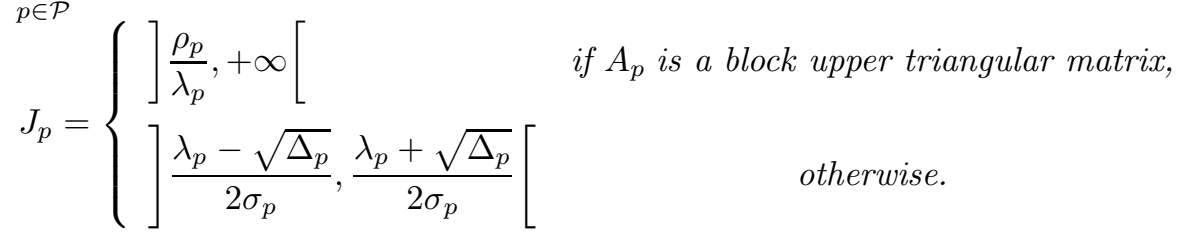

REMARK 3.6. Notice that, for $2 \times 2$-block upper triangular matrices, conditions (1) and (2) of Corollary 3.5 are always satisfied. Therefore, in this case, Corollary 3.5 reduces to stating that the existence of a CQLF for the diagonal block sets guarantees simultaneous stability, in accordance to what is claimed in Theorem 2.2.

4. The $r$-block case. In this section, we show how recursively to apply Corollary 3.5 to the case where the matrices are partitioned into more than two blocks. 
This is useful for instance when the matrices are of larger order and an adequate partition into smaller blocks allows to more easily determine the existence of a CLS for each set of diagonal blocks by using some known results, for instance for blocks of order 2 , see $[12,14,16]$.

Suppose that the matrices $\left\{A_{1}, A_{2}, \ldots, A_{N}\right\}$ are partitioned into $r \times r$ blocks such that each set of diagonal blocks $\mathcal{A}_{i}$ is simultaneous stable with respect to the CLS $P_{i}$, $i=1, \ldots, r$. In order to determine, if a CLS of the form $P=\operatorname{diag}\left(P_{1}, \epsilon_{2} P_{2}, \ldots, \epsilon_{r} P_{r}\right)$ exists, we proceed as follows. First take the $2 \times 2$ left corner of block matrices $A_{p}$, i.e,

$$
A_{p}^{(2)}=\left[\begin{array}{l|l}
A_{11}^{p} & A_{12}^{p} \\
\hline A_{21}^{p} & A_{22}^{p}
\end{array}\right]
$$

and, if possible, by applying Corollary 3.5, determine the positive scalar $\epsilon_{2}$ such that $P^{(2)}=\operatorname{diag}\left(P_{1}, \epsilon_{2} P_{2}\right)$ is a CLS for $A_{p}^{(2)}, p \in \mathcal{P}$. Second take the $3 \times 3$ left corner block of the matrices $A_{p}$, i.e.,

$$
A_{p}^{(3)}=\left[\begin{array}{c|c}
A_{p}^{(2)} & * \\
\hline * & A_{33}^{p}
\end{array}\right]
$$

and, if possible, by applying Corollary 3.5, determine the positive scalar $\epsilon_{3}$ such that $P^{(3)}=\operatorname{diag}\left(P^{(2)}, \epsilon_{3} P_{3}\right)$ is a CLS for $A_{p}^{(3)}$. This procedure is carried out, if possible, until reaching $A_{p}^{(r)}$, which coincides with $A_{p}$, for all $p \in \mathcal{P}$. This amounts to applying Corollary 3.5, if its conditions are satisfied, $r-1$ times. Notice that, this procedure is equivalent to sequentially calculate, when possible, positive scalars $\epsilon_{2}, \ldots, \epsilon_{r}$ such that the positive definiteness of the principal submatrices of

$$
M_{p}=\left[\begin{array}{c|c|cc}
Q_{1}^{p} & -\epsilon_{2}\left(A_{21}^{p}\right)^{T} P_{2}-P_{1} A_{12}^{p} & \ldots-\epsilon_{r}\left(A_{r 1}^{p}\right)^{T} P_{r}-P_{1} A_{1 r}^{p} \\
\cline { 1 - 2 } & \epsilon_{2} Q_{2}^{p} & \ldots-\epsilon_{r}\left(A_{r 2}^{p}\right)^{T} P_{r}-P_{2} A_{2 r}^{p} \\
\vdots \vdots & \vdots & \ddots & \vdots \\
-\epsilon_{r} P_{r} A_{r 1}^{p}-\left(A_{1 r}^{p}\right)^{T} P_{1} & -\epsilon_{r} P_{r} A_{r 2}^{p}-\left(A_{2 r}^{p}\right)^{T} P_{1} P_{2} & \cdots & \epsilon_{r} Q_{r}^{p}
\end{array}\right]
$$

is guaranteed, for all $p \in \mathcal{P}$, being $Q_{i}^{p}=\mathcal{L}_{A_{i i}^{p}}\left(P_{p}\right)>0$.

An algorithm. Based on the given arguments we present an algorithm that enables the identification of a CLS for $r$-block matrices with $r \geq 2$. The algorithm starts from CLSs $P_{1}, P_{2}, \ldots, P_{r}$ for the diagonal blocks and computes, if possible according with the previous arguments, positive scalars $\epsilon_{2}, \ldots, \epsilon_{r}$ such that $P=$ $\operatorname{diag}\left(P_{1}, \epsilon_{2} P_{2}, \ldots, \epsilon_{r} P_{r}\right)$ is a CLS for $\mathcal{A}=\left\{A_{p}: p \in \mathcal{P}\right\}$.

Initiation step $k=2, \quad Q_{1}^{p}=-\left(A_{11}^{p}\right)^{T} P_{1}-P_{1} A_{11}^{p}$ and $M^{p}\left(\epsilon_{1}\right):=Q_{1}^{p}$. 
Step 1 For all $p \in \mathcal{P}$, take $Q_{k}^{p}=-\left(A_{k k}^{p}\right)^{T} P_{k}-P_{k} A_{k k}^{p}$ and

$$
\begin{aligned}
& V_{k}^{p}=\left[\begin{array}{lll}
P_{k} A_{k 1}^{p} & P_{k} A_{k 2}^{p} \cdots & P_{k} A_{k k-1}^{p}
\end{array}\right] \\
& U_{k}^{p}=\left[\begin{array}{llll}
\left(A_{1 k}^{p}\right)^{T} P_{1} & \left(A_{2 k}^{p}\right)^{T} P_{2} & \cdots & \left(A_{k-1 k}^{p}\right)^{T} P_{k-1}
\end{array}\right] \\
& L_{k}^{p}=Q_{k}^{p}-U_{k}^{p}\left[M^{p}\left(\epsilon_{k-1}\right)\right]^{-1}\left(V_{k}^{p}\right)^{T}-V_{k}^{p}\left(M^{p}\left(\epsilon_{k-1}\right)\right)^{-1}\left(U_{k}^{p}\right)^{T} \\
& S_{k}^{p}=V_{k}^{p}\left(M^{p}\left(\epsilon_{k-1}\right)\right)^{-1}\left(V_{k}^{p}\right)^{T} \\
& R_{k}^{p}=U_{k}^{p}\left(M^{p}\left(\epsilon_{k-1}\right)\right)^{-1}\left(U_{k}^{p}\right)^{T}
\end{aligned}
$$

and

$$
\lambda_{k}^{p}=\lambda_{\min }\left(L_{k}^{p}\right), \sigma_{k}^{p}=\lambda_{\max }\left(S_{k}^{p}\right) \text { and } \rho_{k}^{p}=\lambda_{\max }\left(R_{k}^{p}\right) .
$$

Step 2 If $\left(\lambda_{\min }\left(L_{k}^{p}\right)\right)^{2}-4 \lambda_{\min }\left(S_{k}^{p}\right) \lambda_{\min }\left(R_{k}^{p}\right)>0$, for all $p \in \mathcal{P}$, go to Step 3. Else, stop and write "there is no CLS for $\mathcal{A}$ of the form $P=\operatorname{diag}\left(P_{1}, \epsilon_{2} P_{2}, \ldots, \epsilon_{r} P_{r}\right)$ ".

Step 3 Take $\Delta_{k}^{p}:=\left(\lambda_{k}^{p}\right)^{2}-4 \rho_{k}^{p} \sigma_{k}^{p}$. If $\Delta_{k}^{p}>0$, for all $p \in \mathcal{P}$, go to Step 4. Else, stop and write "nothing can be concluded about the existence of a block diagonal CLS".

Step 4 Take for each $p$,

$$
] a_{k}^{p}, b_{k}^{p}\left[= \begin{cases}\frac{\lambda_{k}^{p}-\sqrt{\Delta_{k}^{p}}}{2 \sigma_{k}^{p}}, \frac{\lambda_{k}^{p}+\sqrt{\Delta_{k}^{p}}}{2 \sigma_{k}^{p}}[, & \text { if } V_{k}^{p} \neq 0, \\ \frac{\rho_{k}^{p}}{\lambda_{k}^{p}},+\infty[, & \text { if } V_{k}^{p}=0,\end{cases}\right.
$$

and calculate $a_{k}:=\max \left\{a_{k}^{p}: p \in \mathcal{P}\right\}$ and $b_{k}:=\min \left\{b_{k}^{p}: p \in \mathcal{P}\right\}$. If $a_{k}>b_{k}$, stop and write "nothing can be concluded about the CLS existence". Else, go to Step 5 .

Step 5 Take any $\left.\epsilon_{k} \in\right] a_{k}, b_{k}[$ and consider

$$
M^{p}\left(\epsilon_{k}\right)=\left[\begin{array}{cc}
M^{p}\left(\epsilon_{k-1}\right) & -\epsilon_{k}\left(V_{k}^{p}\right)^{T}-\left(U_{k}^{p}\right)^{T} \\
-\epsilon_{k} V_{k}^{p}-U_{k}^{p} & \epsilon_{k} Q_{k}^{p}
\end{array}\right] .
$$

Step 6 If $k+1>r$, go to Step 9. Else, go to Step 7 .

Step 7 Set $k:=k+1$, until $k=r$.

Step 8 Return to Step 1.

Step 9 Write " $\mathcal{A}$ has a block diagonal CLS of the form $P=\operatorname{diag}\left(P_{1}, \epsilon_{2} P_{2}, \ldots, \epsilon_{r} P_{r}\right)$ ".

ExAmple 4.1. Consider the matrix

$$
A_{1}=\left[\begin{array}{cc|c}
-1 & 0 & -1 \\
0 & -1 & -1 \\
\hline-0.1995 & -0.1 & -0.3
\end{array}\right] .
$$


Note that $P_{1}=I_{2}$ and $P_{2}=1$ are Lyapunov solutions for the diagonal blocks. However, by applying the algorithm, it is easy to conclude that $A_{1}$ does not have a Lyapunov solution of the form $P=\operatorname{diag}\left(I_{2}, \epsilon_{2}\right), \epsilon_{2}>0$. In fact, the condition of Step 2 is not fulfilled since we have $L_{2}^{1} \approx 0.3005, R_{2}^{1}=1$ and $S_{2}^{1} \approx 0.0249$, so

$$
\lambda_{\min }\left(L_{2}^{1}\right)-2\left(\lambda_{\min }\left(R_{2}^{1}\right) \lambda_{\min }\left(S_{2}^{1}\right)\right)^{\frac{1}{2}} \approx-0.0093<0 .
$$

Example 4.2. Consider the matrices

$$
A_{1}=\left[\begin{array}{cc|cc}
-1 & 1 & 1 & -0.5 \\
-1 & -1 & 0 & 2 \\
\hline 9 & -1 & -20 & 2 \\
-0.1 & -1 & -2 & -30
\end{array}\right] \text { and } A_{2}=\left[\begin{array}{cc|cc}
-1 & 1 & 1 & -0.5 \\
-1 & -1 & 0 & 2 \\
\hline 5 & 0 & -20 & -10 \\
-0.1 & -1 & 19 & -11
\end{array}\right]
$$

Note that $P_{1}=I_{2}$ and $P_{2}=\operatorname{diag}(1,2.09)$ are CLSs for the diagonal blocks $(1,1)$ and $(2,2)$, respectively. Let us use the algorithm in order to compute, if possible, a CLS of the form $P=\operatorname{diag}\left(I_{2}, \epsilon_{2} P_{2}\right)$, where $\epsilon_{2}>0$, for $\mathcal{A}=\left\{A_{1}, A_{2}\right\}$. For the Initiation step, we have $M^{1}\left(\epsilon_{1}\right)=Q_{1}^{1}=\operatorname{diag}(2,2)$ and $M^{2}\left(\epsilon_{1}\right)=Q_{1}^{2}=\operatorname{diag}(2,2)$. In Step 1, we

\begin{tabular}{|c|c|c|c|c|c|}
\hline & $V_{2}^{p}$ & $U_{2}^{p}$ & $L_{2}^{p}$ & $S_{2}^{p}$ & $R_{2}^{p}$ \\
\hline \multirow{3}{*}{$A_{1}$} & $\begin{array}{ll}9 & -1\end{array}$ & 10 & $31 \quad 5.535$ & $41 \quad 0.105$ & $0.5-0.25$ \\
\hline & $-0.209-2.09$ & $\lfloor-0.52]$ & 5.535129 .476 & 0.1052 .206 & {$[-0.252 .125$} \\
\hline & & & $\lambda_{2}^{1} \approx 30.699$ & $\sigma_{2}^{1} \approx 41$ & $\rho_{2}^{1} \approx 2.163$ \\
\hline \multirow{3}{*}{$A_{2}$} & 0 & $\left.\begin{array}{ll}1 & 0\end{array}\right]$ & $35 \quad-28.356$ & $12.5-0.523$ & $0.5-0.25$ \\
\hline & L-0.209-2.09 & $\lfloor-0.52\rfloor$ & L-28.356 50.056 & {$\left[\begin{array}{ll}-0.523 & 2.206\end{array}\right.$} & L-0.25 2.125 \\
\hline & & & $\lambda_{2}^{2}=13.19$ & $\sigma_{2}^{2} \approx 12.527$ & $\rho_{2}^{2} \approx 2.163$ \\
\hline
\end{tabular}
obtain $Q_{2}^{1}=\left[\begin{array}{cc}40 & 2.18 \\ 2.18 & 125.4\end{array}\right]$ and $Q_{2}^{2}=\left[\begin{array}{cc}9 & -1 \\ -0.209 & -2.09\end{array}\right]$. Moreover,

Since the conditions of Step 3 are satisfied, we compute the intervals of Step 4:

$$
\left.J_{1} \approx\right] 0.079,0.670\left[\text { and } J_{2} \approx\right] 0.203,0.850[\text {. }
$$

Taking $\epsilon_{2} \in J_{1} \cap J_{2}$, for example $\epsilon_{2}=0.5$, we conclude that $A_{1}$ and $A_{2}$ share $P=$ $\operatorname{diag}(1,1,0.5,1.045)$ as a CLS.

5. Conclusion. In this work, we have investigated the block simultaneous diagonal stability of a collection $\mathcal{A}=\left\{A_{p}: p \in \mathcal{P}\right\}$ of matrices partitioned into $r \times r$ blocks, i.e. $A_{p}=\left[A_{i j}^{p}\right]$, for $i, j=1, \ldots, r$, where, for each $i$ the diagonal blocks $A_{i i}^{p}$ are square matrices of the same size, for all $p \in \mathcal{P}$. It turns out that a necessary condition for $\mathcal{A}$ to be a $r$-block simultaneous block diagonally stable is that each set 
of diagonal blocks $\mathcal{A}_{i}=\left\{A_{i i}^{p}: p \in \mathcal{P}\right\}$ should be simultaneous stable, that is, it must have a common Lyapunov matrix solution $P_{i}$. Under this assumption, we have proposed a constructive method to obtain a common Lyapunov matrix solution with block diagonal structure for $\mathcal{A}$ with diagonal blocks depending on $P_{i}, i=1, \ldots, r$, if certain conditions hold, Theorem 3.3 and Corollary 3.5. These results generalize the one obtained in [5] for the block upper triangular case. Notice that with our approach we may deal with the diagonal stability itself by considering the diagonal elements as blocks of order 1. In particular case of matrices of order two, the sufficient conditions given by Theorem 3.3 are equivalent to the classical characterization of diagonal stability [6].

Acknowledgment. This work was supported by FEDER funds through COMPETE-Operational Programme Factors of Competitiveness ("Programa Operacional Factores de Competitividade") and by Portuguese funds through the Center for Research and Development in Mathematics and Applications (University of Aveiro) and the Portuguese Foundation for Science and Technology ("FCT-Fundação para a Ciência e a Tecnologia"), within project PEst-C/MAT/UI4106/2011 with COMPETE number FCOMP-01-0124-FEDER-022690.

\section{REFERENCES}

[1] T. Ando. Set of matrices with common Lyapunov solution. Archiv der Mathematik, 77:76-84, 2001.

[2] M. Arcak. Diagonal stability on cactus graphs and application to network stability analysis. IEEE Transactions on Automatic Control, 56(12):2766-2777, 2011.

[3] I. Brás, A. Carapito, and P. Rocha. Stability of interconnected switched systems. Proceedings of the 7th Portuguese Conference on Automatic Control, 2006.

[4] T. Büyükköroğlu. Common diagonal lyapunov function for third order linear switched system. Journal of Computational and Applied Mathematics, 236:3647-3653, 2012.

[5] D. Cheng, L. Guo, and J. Huang. On quadratic Lyapunov functions. IEEE Transactions on Automatic Control, 48:885-890, 2003.

[6] G.W. Cross. Three types of matrix stability. Linear Algebra and its Applications, 20:253-263, 1978.

[7] Z. Guisheng and X. Xuping. A unified approach to stability analysis of switched linear descriptor systems under arbitrary switching. International Journal of Applied Mathematics and Computer Science, 20(2):249-259, 2010.

[8] R.A. Horn and C.R. Johnson. Matrix Analysis. Cambridge University Press, 1985.

[9] E. Kaszkurewicz and A. Bhaya. Matrix Diagonal Stability in Systems and Computation. Birkhäuser, Boston, 2000.

[10] T.J. Laffey and H. Šmigoc. Sufficient conditions on commutators for a pair of stable matrices to have a common solution to the Lyapunov equation. SIAM Journal on Matrix Analysis and Applications, 31:2017-2028, 2010.

[11] D. Liberzon. Switching in Systems and Control. Birkhauser, Boston, 2003.

[12] D. Liberzon, J.P. Hespanha, and A.S. Morse. Stability of switched systems: a lie-algebraic condition. Systems Control Letters, 37(3):117-122, 1999. 
[13] D. Liberzon and A.S. Morse. Basic problems in stability and design of switched systems. IEEE Control Systems, 19:59-70, 1999.

[14] K.S. Narendra and J. Balakrishnan. A common Lyapunov function for stable LTI systems with commuting $\mathcal{A}$-matrices. IEEE Transactions on Automatic Control, 39(4):2469-2471, 1994.

[15] S. Roy and A. Saberi. On structural properties of the Lyapunov matrix equation for optimal diagonal solutions. IEEE Transactions on Automatic Control, 54:2234-2238, 2009.

[16] R.N. Shorten and K.S. Narendra. Necessary and sufficient condictions for the existence of a commom quadratic lyapunov function for a finite number of stable second order linear time-invariant systems. International Journal of Adaptive Control and Signal Processing, 16:709-728, 2002.

[17] Robert Shorten, et al. Stability criteria for switched and hybrid systems. SIAM Review, 49(4):545-592, 2007. 